\title{
Effects of one-week tongue-task training on sleep apnea severity: A pilot study
}

\author{
Eric Rousseau MSc ${ }^{1}$, César Augusto Melo-Silva $\mathrm{PhD}^{1,2}$, Simon Gakwaya MSc ${ }^{1}$, Frédéric Sériès MD ${ }^{1}$
}

E Rousseau, CA Melo-Silva, S Gakwaya, F Sériès. Effects of one week tongue-task training on sleep apnea severity: A pilot study. Can Respir J 2015;22(3):176-178.

The aim of the present study was to assess the effects of one-week tonguetask training (TTT) on sleep apnea severity in sleep apnea subjects. Ten patients with sleep apnea (seven men, mean [ \pm SD] age $52 \pm 8$ years; mean apnea-hypopnea $[\mathrm{AHI}]$ index $20.9 \pm 5.3$ events/h) underwent $1 \mathrm{~h}$ TTT in the authors' laboratory on seven consecutive days. A complete or limited recording and tongue maximal protruding force were assessed before and after one-week TTT. One-week TTT was associated with a global AHI decrease (pre-TTT: 20.9 \pm 5.3 events/h; post-TTT: $16.1 \pm 5.1$ events $/ \mathrm{h}$; $\mathrm{P}<0.001$ ) and AHI decrease during rapid eye movement sleep (pre-TTT: $32.2 \pm 18.4$ events/h; post-TTT: $16.7 \pm 6.6$ events $/ \mathrm{h} ; \mathrm{P}=0.03$ ), while protruding force remained unchanged. The authors consider these results to be potentially clinically relevant and worthy of further investigation in a large randomized trial.

Key Words: Exercise; Obstructive sleep apnea; Rehabilitation; Sleep-disordered breathing

$\mathrm{O}$ bstructive sleep apnea-hypopnea syndrome (SAHS) is characterized by repetitive episodes of upper airway (UA) closure. SAHS is associated with altered quality of life, and increased cardiovascular and metabolic morbidity and mortality. The prevalence of SAHS is as high as $24 \%$ in men and $9 \%$ in women (1). UA closure occurs when dilating forces cannot overcome the collapsing effect of the negative transmural inspiratory pressure gradient and tissue weight. The amount and timing of neuromuscular activation of UA stabilizing muscles, their propensity to fatigue and their mechanical efficiency are pivotal in maintaining UA stability (2). Furthermore, the sleepinduced decrease in tonic and phasic UA activity has a detrimental impact on the maintenance of UA patency and stability.

A lack of efficiency in UA stabilizing muscle contraction makes them prone to fatigue and, thus, further alters their ability to maintain UA patency. Short-term genioglossus (GG) activation with $10 \mathrm{~min}$ of moderate UA resistance loading during mild hypercapnia is accompanied by an approximately $50 \%$ decrease in GG endurance (3). In vitro studies have demonstrated that in nonobese obstructive sleep apnea (OSA) patients, GG fatigue improved after continuous positive pressure airway therapy (4). More recently, a decrease in endurance time in response to repeated, short-duration, voluntary isometric contraction was found to be the only significant physiological feature differentiating sensorimotor characteristics between individuals with OSA and snorers (5). Consistent with such observations, individuals with OSA demonstrated a greater decrease in GG contraction velocity than controls during sustained isometric tongue protrusion (6). The role of fatigue in the worsening of obstructive breathing disorders throughout the night is further supported by our recent results obtained using a phrenic nerve magnetic stimulation model, showing that the decline in UA stability following UA pressure loading during wakefulness correlates with increments of the apnea-hypopnea index (AHI) from the first to the final quarter of the night (7).

\author{
Projet-pilote sur les effets d'un entraînement d'une \\ semaine pour positionner la langue sur la gravité de \\ l'apnée du sommeil
}

La présente étude visait à évaluer les effets d'un entrainement d'une semaine pour positionner la langue (EPL) sur la gravité de l'apnée du sommeil chez des sujets atteints de ce trouble. Pendant sept jours consécutifs, dix patients atteints d'apnée du sommeil (sept hommes, âge moyen [ \pm ÉT] de $52 \pm 8$ ans; indice moyen d'apnée-hypopnée [IAH] de 20,9 $\pm 5,3$ événements/h) se sont soumis à un EPL d'une heure au laboratoire des auteurs. Les chercheurs ont procédé à un enregistrement complet ou partiel des patients et ont évalué la force maximale de saillie de la langue avant et après la semaine d'EPL. L'EPL d'une semaine s'associait à une diminution globale de l'IAH (avant l'EPL : 20,9 95,3 événements/h; après l'EPL: 16,1 \pm 5 , 1 événements/h; $\mathrm{P}<0,001)$ et à une diminution de l'IAH pendant le sommeil paradoxal (avant l'EPL : 32,2 $\pm 18,4$ événements/h; après l'EPL : 16,7 $\pm 6,6$ événements/h; $\mathrm{P}=0,03$ ), tandis que la force de saillie demeurait inchangée. Les auteurs pensent que ces résultats pourraient avoir une pertinence clinique et méritent d'être examinés dans le cadre d'un grand essai aléatoire.

Thus, there is some evidence that UA muscle fatigue is a key factor of SAHS pathophysiology and, as such, represents a potentially important physiological trait. Improving this trait by enhancing activity/efficacy of UA muscles may represent a new, effective treatment approach for SAHS.

Different noninvasive brain stimulation modalities can be used to modify corticomotor excitability, including different stimulation techniques such as repetitive transcranial magnetic stimulation (rTMS). rTMS applied over the supplementary motor area enhances the corticomotor response of the diaphragm (8). Enhancing corticomotor excitability also has mechanical consequences, as indicated by a decline in the loss of maximal voluntary force developed by the first interosseous muscle during a fatigue protocol (9). Improvement in synergistic muscle coupling following noninvasive brain stimulation could be involved in such mechanical effects. We are aware of only one SAHS study investigating changes in corticomotor excitability after rTMS (10). The motor threshold of the first dorsal interosseus muscle decreased similarly in SAHS and controls, but the increase in motor-evoked potential amplitude was lower in SAHS.

Thus, given that increasing corticomotor excitability improves the mechanical performance of the targeted muscle, we reasoned that clinical strategies aimed at increasing GG corticomotor excitability should represent easy, practical and efficient ways to improve UA stability and sleep-disordered breathing. It was reported that tongue-task training (TTT), consisting of generating low-intensity isometric tongue protrusion force for $1.5 \mathrm{~s}$ every $10 \mathrm{~s}$ during a $1 \mathrm{~h}$ session on seven consecutive days, was associated with a $50 \%$ increase in GG corticomotor excitability and an increase in cortical motor area that persisted for one week after the end of the training protocol (11). Thus, the aim of the present study was to assess the effects of one-week TTT on tongue protusion force and sleep apnea severity in sleep apnea subjects.

${ }^{1}$ Unité de recherche en pneumologie, Centre de recherche, Institut Universitaire de Cardiologie et de Pneumologie de Québec (IUCPQ), Université Laval, Québec, Québec; ${ }^{2}$ Laboratory of Respiratory Physiology, University of Brasília, UnB, Brasília, DF, Brazil

Correspondence: Dr Frédéric Sériès, Centre de recherche, IUCPQ, 2725 chemin Sainte-Foy, Québec, Québec G1V 4G5.

Telephone 418-656-4747, fax 418-656-4762, e-mail frederic.series@fmed.ulaval.ca 


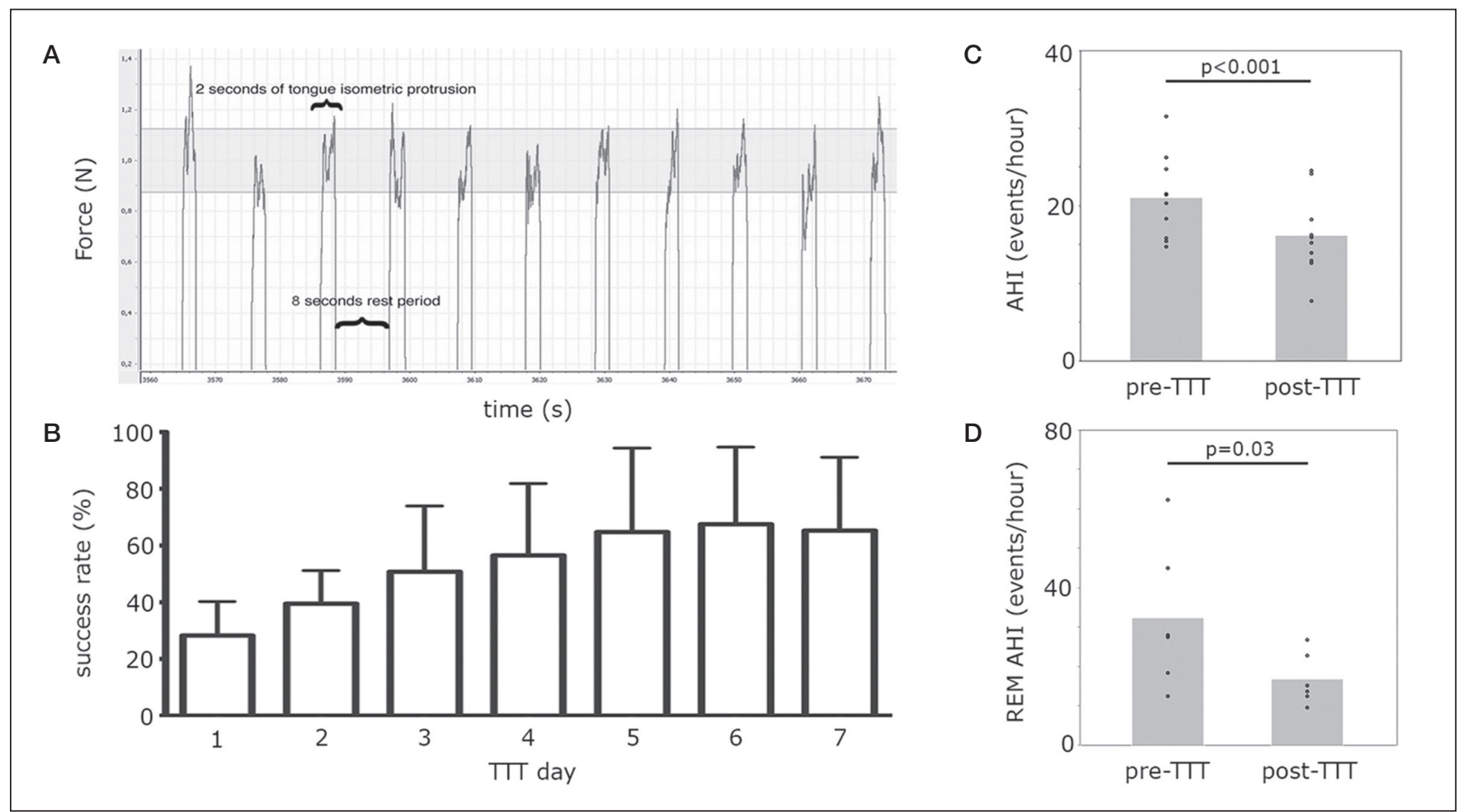

Figure 1) A Example of the force signal profile during tongue-task training (TTT), in which a constant tongue protusion force of $1.0 \mathrm{~N}$ is held for $2 \mathrm{~s}$ alternating with an $8 \mathrm{~s}$ rest period. The grey area represents the preset force target window. B Success rate (mean \pm SD) from first to seventh day of the TTT. $\mathrm{C}$ Mean apnea-hypopnea index (AHI) pre-and post-TTT during entire sleep night $(n=10)$. D Mean AHI pre-and post-TTT during rapid-eye movement (REM) sleep $(n=6)$

\section{METHODS}

Ten sleep apnea patients (seven men; mean [ \pm SD] age $52 \pm 8$ years; body mass index $26.5 \pm 1.8 \mathrm{~kg} / \mathrm{m}^{2}$; neck circumference $35.6 \pm 3.9 \mathrm{~cm}$;

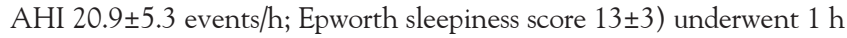
TTT in the laboratory for seven consecutive days. Briefly, the subjects were asked to place their tongue onto a force transducer positioned below the incisors. The protusion force is displayed on a computer screen positioned in front of the subject. During each tongue-protusion trial, they were asked to generate a force of $1.0 \pm 0.12 \mathrm{~N}$ by maintaining the force display inside the corresponding preset force target window. The TTT consisted of maintaining the force inside the target window for $2 \mathrm{~s}$ alternating with a rest period of approximately $8 \mathrm{~s}(10 \mathrm{~s}$ task period; 360 trials [Figure 1A]). The proportion of time for which subjects actually maintained the force inside the target window in relation to the total protusion task time (720 s) was defined as the success rate. A sleep recording (in-laboratory or ambulatory) and tongue maximal protuding force (Fmax) were assessed before and immediately after training. In six subjects, the sleep recordings consisted of a conventional in-laboratory polysomnography study. An ambulatory cardiorespiratory recording (Embletta, USA) was completed in four subjects before and after the one-week TTT, which provided AHI and desaturation index; however, specific data regarding sleep characteristics and sleep stage-specific AHI could not be collected. All data were analyzed using a two-tailed Student's paired $t$ test. All analyses were performed using JMP version 10.0 (SAS Institute, USA). The significance level was set at $5 \%$, and the results were expressed as mean \pm SD. The internal review board of the authors' institution approved the protocol and informed consent was obtained from all subjects.

\section{RESULTS}

The TTT procedure was well tolerated in all subjects. The $1.0 \mathrm{~N}$ protusion force targeted during the TTT corresponded to an average of $3.6 \%$ of Fmax. Fmax remained unchanged $(n=10$; pre-TTT: $29.5 \pm 4.9 \mathrm{~N}$; post-TTT: $31.5 \pm 8.3 \mathrm{~N} ; \mathrm{P}=0.3$ ). On average, the success rate improved by $132 \%$ from the beginning to the end of the one-week TTT ( $n=10$; first TTT session: $28 \pm 10 \%$; final TTT session: $65 \pm 21 \%$; $\mathrm{P}=0.0006$ ) (Figure 1B).

During the entire night, AHI decreased by $23 \%(\mathrm{n}=10$; pre-TTT: 20.9 \pm 5.3 events/h; post-TTT: $16.1 \pm 5.1$ events $/ \mathrm{h} ; \mathrm{P}<0.001$ ) (Figure $1 \mathrm{C}$ ) and progressed from a moderate to mild apnea severity in $40 \%$ of patients, in which mild and moderate apnea are characterized, respectively, by an AHI between 5 to 15 events/h and 15 to 30 events/h of sleep, according to the American Academy of Sleep Medicine (12). During rapid-eye movement (REM) sleep, the AHI decreased by $48 \%$ $(\mathrm{n}=6$; pre-TTT: $32.2 \pm 18.4$ events/h; post-TTT: $16.7 \pm 6.6$ events $/ \mathrm{h}$; $\mathrm{P}=0.03$ ) (Figure 1D) with also a borderline significant decrease during non-REM sleep $(n=6$; pre-TTT: $16.1 \pm 3.1$ events/h; post-TTT: $12.9 \pm 4.3$ events $/ h ; P=0.07)$. However, there were no changes in desaturation index $(\mathrm{n}=10$; pre-TTT: $9.1 \pm 7.5$ events/h; post-TTT: $7.1 \pm 5.1$ events/h; $\mathrm{P}=0.19)$, arousal index $(\mathrm{n}=6$; pre-TTT: $12.0 \pm 1.5$ events $/ \mathrm{h}$; post-TTT: $11.4 \pm 3.0$ events/h; $\mathrm{P}=0.72)$ and sleep efficiency $(\mathrm{n}=6$; pre-TTT: $86 \pm 12 \%$; post-TTT: $87 \pm 10 \%$; $\mathrm{P}=0.70$ ). The total sleep time also remained unchanged $(n=10$; pre-TTT: $414 \pm 55$ s; post-TTT: $443 \pm 69$ s; $\mathrm{P}=0.10)$.

\section{DISCUSSION}

It is noteworthy that our results are consistent with the findings from a three-month oropharyngeal exercise protocol in which daily $1 \mathrm{~h}$ exercises induced a decrease in AHI only during REM sleep (13). It emphasizes that the improvement in AHI during REM sleep following one-week TTT was similar to that observed following the threemonth exercise protocol. This suggests that one-week TTT is sufficient to confer benefits; therefore increasing the interest for a clinical application. Another argument supporting a short-term benefit is the improvement in success rate, which appears to have reached a plateau within the one-week time frame (Figure 1B). The absence of change in Fmax is not surprising because the TTT protocol is not oriented toward strength gain, but rather toward an enhancement in GG 
cortical excitability and improvement in the coordination of the GG protusion task. There is a need to explore how long these improvements persist after the cessation of the exercises and how they can improve the development of GG muscle fatigue.

We acknowledge that, as a pilot study, a limitation of our analysis was that it was a before-and-after single-arm study, without control a group; therefore, we cannot exclude the possibility of a regression toward the mean.

\section{CONCLUSION}

One-week TTT was associated with a $48 \%$ decrease in obstructive breathing disturbances during REM sleep. We consider these results to be clinically relevant and worthy of further investigation in a controlled study, in which changes in nocturnal breathing disorders, in neurophysiological and mechanical features of UA stabilizing muscles and their time course would be documented.

\section{REFERENCES}

1. Young T, Palta M, Dempsey J, Skatrud J, Weber S, Badr S. The occurrence of sleep-disordered breathing among middle-aged adults. N Engl J Med 1993;328:1230-5.

2. Bilston LE, Gandevia SC. Biomechanical properties of the human upper airway and their effect on its behavior during breathing and in obstructive sleep apnea. J Appl Physiol 2014;116:314-24.

3. Scardella AT, Krawciw N, Petrozzino J, Co MA, Santiago TV, Edelman NH. Strength and endurance characteristics of the normal human genioglossus. Am Rev Respir Dis 1993;148:179-84.

4. Carrera M, Barbé F, Sauleda J, Tomás M, Gómez C, Agustí AG. Patients with obstructive sleep apnea exhibit genioglossus dysfunction that is normalized after treatment with continuous positive airway pressure. Am J Respir Crit Care Med 1999;159:1960-6

5. Eckert DJ, Lo YL, Saboisky JP, Jordan AS, White DP, Malhotra A. Sensorimotor function of the upper-airway muscles and respiratory sensory processing in untreated obstructive sleep apnea. J Appl Physiol 2011;111:1644-53.

6. McSharry D, O'Connor C, McNicholas T, et al. Genioglossus fatigue in obstructive sleep apnea. Respir Physiol Neurobiol 2012;183:59-66.

7. Gakwaya S, Ethier G, Sériès F. Influence of negative airway pressure on upper airway dynamic and impact on night-time apnea worsening. Respir Physiol Neurobiol 2012;181:88-94.
ACKNOWLEDGEMENTS \& FUNDING: This work was supported by the Canadian Institutes of Health Research [grant number 89985].

AUTHOR CONTRIBUTIONS: ER contributed to data acquisition, data analysis and interpretation, preparing the figures, drafting the manuscript, review and final approval of the draft. CAMS contributed to data acquisition, data analysis and interpretation, preparing the figures, review and final approval of the draft. SG contributed to data acquisition, data analysis and interpretation, review and final approval of the draft. FS contributed to acquisition of funding, conception and study design, data interpretation, review and final approval of the draft.

DISCLOSURES: The authors have no financial disclosures or conflicts of interest to declare.

8. Adler D, Gottfried SB, Bautin N, et al. Repetitive magnetic stimulation of the phrenic nerves for diaphragm conditioning: A normative study of feasibility and optimal settings. Appl Physiol Nutr Metab 2011;36:1001-8.

9. Benwell NM, Mastaglia FL, Thickbroom GW. Paired-pulse rTMS at trans-synaptic intervals increases corticomotor excitability and reduces the rate of force loss during a fatiguing exercise of the hand. Exp Brain Res 2006;175:626-32.

10. Das A, Anupa AV, Radhakrishnan A. Reduced plastic brain responses to repetitive transcranial magnetic stimulation in severe obstructive sleep apnea syndrome. Sleep Med 2013;14:636-40.

11. Svensson P, Romaniello A, Wang K, Arendt-Nielsen L, Sessle BJ. One hour of tongue-task training is associated with plasticity in corticomotor control of the human tongue musculature. Exp Brain Res 2006;173:165-73.

12. Epstein LJ, Kristo D, Strollo PJ, et al. Clinical guideline for the evaluation, management and long-term care of obstructive sleep apnea in adults. J Clin Sleep Med 2009;5:263-76.

13. Guimarães K, Drager L, Genta P, Marcondes B, Lorezi-Filho G. Effects of oropharyngeal exercices on patients with moderate obstructive sleep apnea syndrome. Am J Respir Crit Care Med 2009;179:962-8. 


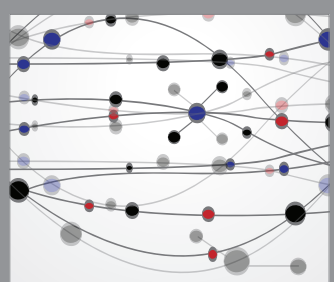

The Scientific World Journal
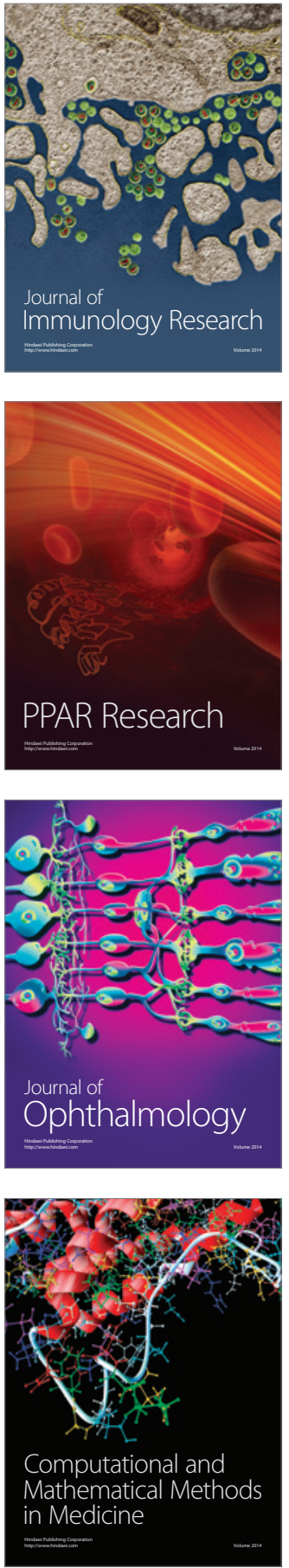

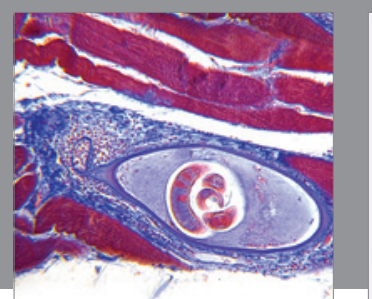

Gastroenterology Research and Practice

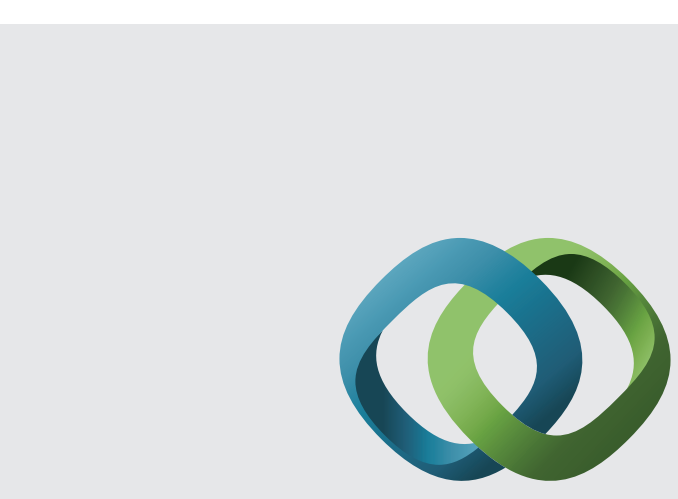

\section{Hindawi}

Submit your manuscripts at

http://www.hindawi.com
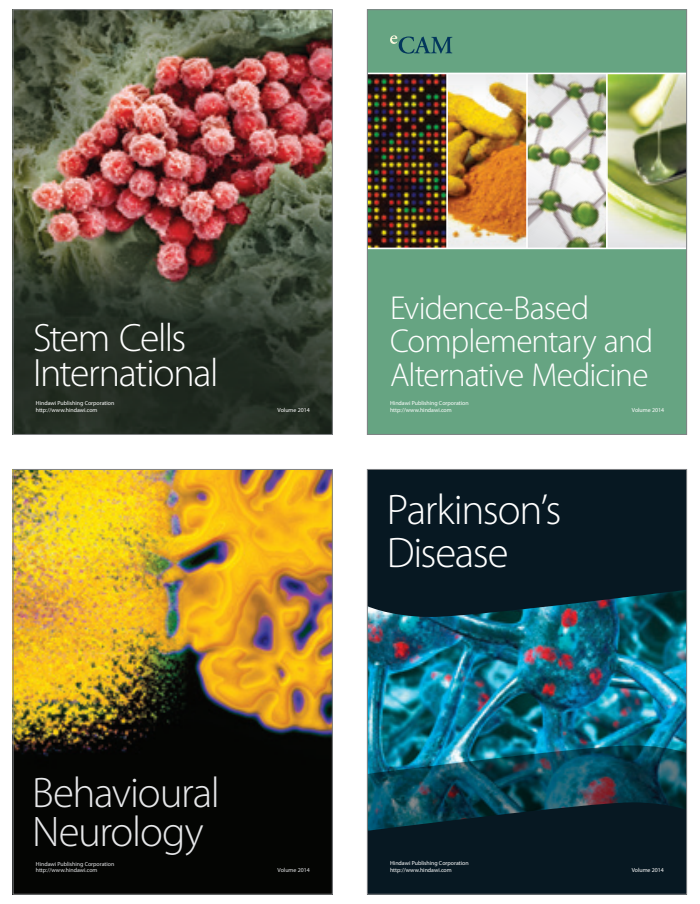
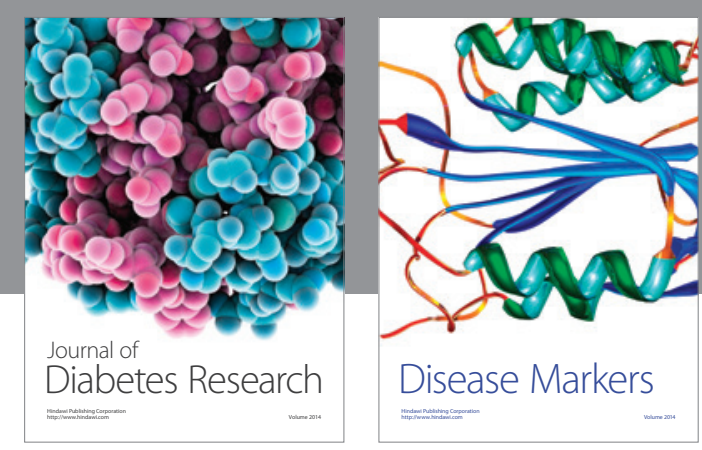

Disease Markers
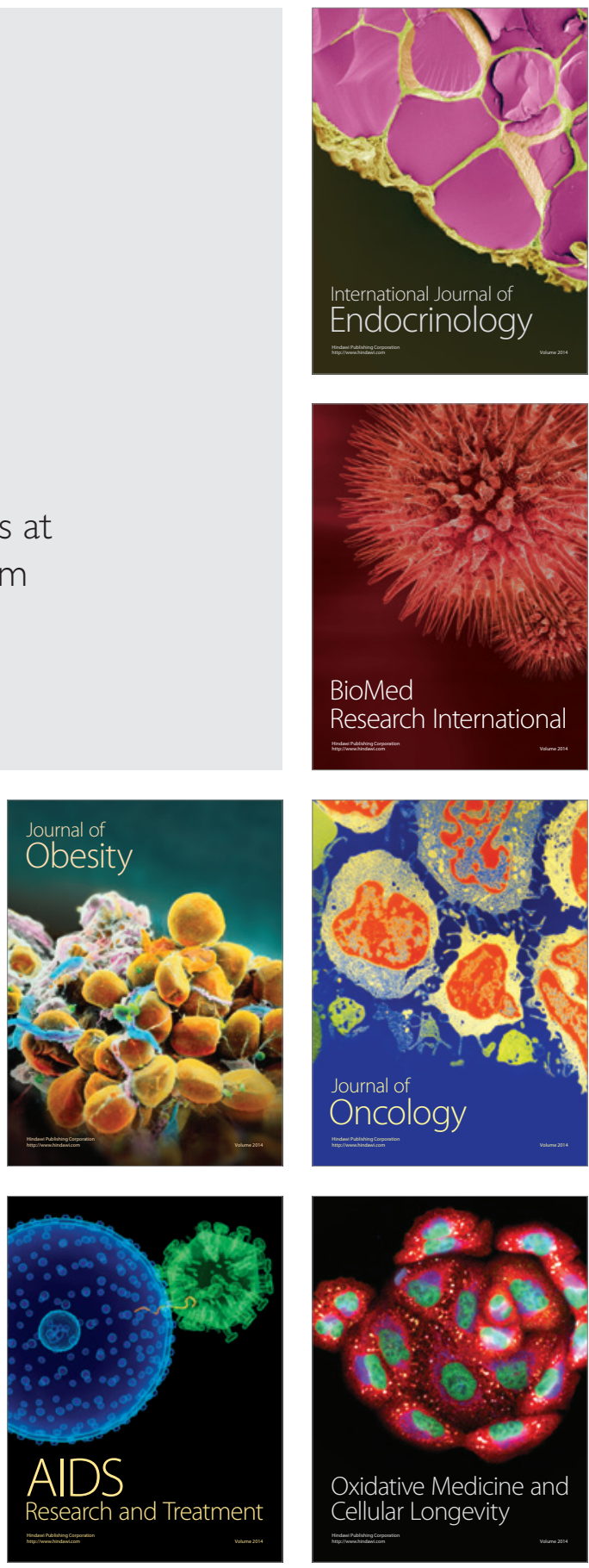